Cahiers $d u$ MONDE RUSSE

\section{Cahiers du monde russe}

Russie - Empire russe - Union soviétique et États indépendants

$55 / 3-4 \mid 2014$

Varia

\title{
Diane P. Koenker, Club Red, Vacation Travel and the Soviet Dream
}

\section{Laurent Coumel}

\section{OpenEdition}

\section{Journals}

Édition électronique

URL : http://journals.openedition.org/monderusse/8102

DOI : $10.4000 /$ monderusse. 8102

ISSN : $1777-5388$

\section{Éditeur}

Éditions de l'EHESS

\section{Édition imprimée}

Date de publication : 1 juillet 2014

Pagination : 464-468

ISBN : 978-2-7132-2441-6

ISSN : $1252-6576$

\section{Référence électronique}

Laurent Coumel, « Diane P. Koenker, Club Red, Vacation Travel and the Soviet Dream », Cahiers du monde russe [En ligne], 55/3-4 | 2014, mis en ligne le 14 avril 2015, Consulté le 24 septembre 2020. URL : http://journals.openedition.org/monderusse/8102; DOI : https://doi.org/10.4000/monderusse. 8102

Ce document a été généré automatiquement le 24 septembre 2020.

(c) École des hautes études en sciences sociales 


\title{
Diane P. Koenker, Club Red, Vacation Travel and the Soviet Dream
}

\author{
Laurent Coumel
}

\section{RÉFÉRENCE}

Diane P. KOENKER, Club Red, Vacation Travel and the Soviet Dream, Ithaca, NY : Cornell University Press, 2013, 307 p.

1 Partant du postulat que « le temps libre était sans doute aussi important que le travail dans la formation de la personnalité soviétique » (p. 1) Diane Koenker, historienne du social, livre une somme ambitieuse sur les « séjours de vacances » en URSS, depuis la fondation jusqu'à la chute du régime. Club Red s'attaque ainsi à deux sortes de pratiques distinctes, en russe otdyh et turizm - le second ayant été récemment analysé par Anne Gorsuch pour la période khrouchtchévienne, en particulier sous l'angle des voyages à l'étranger ${ }^{1}$. Koenker et Gorsuch avaient déjà dirigé il y a quelques années un ouvrage pionnier sur les formes de voyage et de villégiature à l'est de l'Europe ${ }^{2}$.

2 Le repos (otdyh) renvoie au séjour réparateur dans un sanatorium (hérité de la période tsariste et toujours présent dans l'univers soviétique) ou une «maison de repos", moins médicalisée que le premier. Il est défini comme recouvrement des forces, récupération, par un régime qui se veut au service des travailleurs et vise ainsi à entretenir et à accroître leur capacité productive. La construction des infrastructures, le fonctionnement des établissements et surtout la distribution des putevki [bons de séjour] qui permettent d'y accéder reviennent au ministère de la Santé et, de plus en plus, à l'administration centrale des syndicats (renforçant ainsi son rôle de prestataire de services), qui en obtient le quasi-monopole en 1960 : de là sans doute une incapacité durable à répondre à la demande de vacances en famille, les enfants n'étant que progressivement admis, à partir des années 1950, dans certains lieux de séjour. 
Koenker, qui mentionne en passant les camps de pionniers destinés à prendre en charge une partie de la jeunesse, voit dans ce manque un défaut majeur du système, source de frustration pour la population. Gageons toutefois que nombre de parents se satisfaisaient de cette séparation générationnelle et de la possibilité offerte aux adultes de passer quelques semaines seuls, éventuellement libres de rechercher une aventure extraconjugale - un thème récurrent des comédies du cinéma soviétique ayant pour toile de fond un lieu de vacances (kurort). Pendant des décennies, les autorités ont d'abord été soucieuses de renforcer la part des ouvriers dans les statistiques des contingents de pensionnaires, au point d'inclure dans cette catégorie certains cadres d'usine. Elle semble effectivement progresser dans les années 1960, effet de la massification. Mais c'est loin d'être le cas pour les kolkhoziens, qui restent privés de vacances, et une enquête plus fine montre que les destinations les plus prestigieuses sont l'apanage de « l'intelligentsia » ou des « cols blancs », bref, d'une certaine élite (les plus privilégiés, cadres du parti et des ministères, ayant leurs propres lieux de villégiature à part et réservés). Il s'agit des villes thermales du Caucase, de la Crimée et surtout de la côte caucasienne de la mer Noire (Sotchi, destination lancée par Stalin et voulue dès le départ comme une vitrine des vacances soviétiques), mais aussi des navires de croisière sur la Volga et la mer Noire, avant les séjours à l'étranger permis à de rares élus avant 1935, puis à nouveau après 1955 pour des candidats triés sur le volet.

Le « tourisme » connaît un développement quelque peu différent ; le mot désigne dès la fin du XIX ${ }^{e}$ siècle des vacances en mouvement, avec une dimension sportive - à pied, sur l'eau ou sur route (à vélo ou, à partir des années 1930, en voiture individuelle). L'individu y est, du moins dans l'idéal du «tourisme prolétarien » des années 1920, soumis au collectif, groupe d'enthousiastes aspirant à se dépasser pour atteindre un objectif précis : couvrir une distance, accomplir un exploit comme l'ascension d'un sommet, ou simplement vivre ensemble des moments ritualisés (le feu de camp, la quête de nourriture et la préparation du repas, les matchs de volley-ball...). Lors du Grand Tournant stalinien, il est aussi question, dans les publications sur le tourisme, d'apporter la «culture » et la «connaissance » aux contrées éloignées de la modernité soviétique, une mission civilisatrice qui s'estompe ensuite. La dimension "prolétarienne » est, quant à elle, une illusion : le nombre de 1,5 million de membres ouvriers de l'association touristique officielle en 1932 se révèle totalement exagéré ; c'est alors que les syndicats reprennent le contrôle d'infrastructures qui font figure de parent pauvre à côté des luxueux établissements de repos. Cette mainmise est contestée par des "puristes ", "activistes du tourisme " qui s'expriment dans les colonnes du magazine Na suše i na more (Sur la terre ferme et en mer) fondé en 1929, mais Koenker ne s'attarde guère sur ces acteurs ni sur leurs trajectoires. Elle montre en revanche la volonté de contrôle des déplacements sur le territoire intérieur soviétique, illustrée par la campagne contre le "vagabondage" qui vise des touristes non enregistrés (dont peut-être d'authentiques marginaux fuyant les répressions ?) à partir de 1930. Relancé après la guerre, le «tourisme soviétique " s'apparente de plus en plus au scoutisme : les rassemblements (slety) organisés à l'échelle régionale ou républicaine sont l'occasion de compétitions diverses. Dans ces mêmes années 1950, un tournant s'opère dans les attentes des citoyens à l'égard de leurs vacances : de « répit vis-à-vis de la routine du travail », elles deviennent aspiration à « un monde merveilleux » (p. 136), une forme d'évasion de la réalité. D'où, avec l'amélioration des transports intérieurs et du pouvoir d'achat des citadins, le succès impressionnant du tourisme. En 1964 il 
concerne 3 millions de Soviétiques, dont plus des trois quarts voyagent de façon indépendante, "non organisée », tout en profitant des services offerts par les «bases touristiques ", campings où l'on peut obtenir l'insigne officiel de « touriste d'URSS » au terme d'une épreuve strictement définie. En 1980, ce sont plus de 22 millions de personnes qui passent par les turbazy alors que 17 millions d'autres ont profité d'un séjour en maison de repos ou sanatorium. S'ajoutent 50 millions de départs en randonnée le week-end ou pour la journée enregistrés en 1974. Koenker s'intéresse à la tentation chez plusieurs acteurs de mettre en place des mécanismes de marché pour l'octroi des putevki touristiques (non pris en charge par les syndicats, les citoyens payant de leur poche ce type de vacances) - mais elle ne s'attarde guère sur les pratiques de piston et de marché noir qui expliquent que la plupart des patients des maisons médicalisées n'ont aucunement besoin de traitement, comme le déplorent les rapports officiels de façon récurrente dès les années 1920. Le recours à la publicité semble une concession à la loi de l'offre et de la demande dans cette société de consommation soviétique qui s'affirme sous Hruščev. Koenker parle d'un tourisme «postprolétarien» (chapitre 6), la formule renvoyant au triomphe de la «classe moyenne» (p. 166) et de ses valeurs: "une utopie construite sur le savoir et sur la prospérité, exprimée dans les loisirs autant que dans le travail » (p. 211). Son idéal est résumé par un ingénieur répondant à une enquête en 1966 : «C'est tout simplement merveilleux quand des gens de professions, goûts, caractères et niveaux culturels différents se mettent ensemble dans un collectif ». Les pratiques demeurent pourtant compartimentées, y compris par genres: des études révèlent que les femmes célibataires soviétiques prisent davantage les voyages organisés, en particulier en Europe de l'Est et dans les grandes villes, alors que les hommes préfèrent la campagne, et que de plus en plus de "touristes" préfèrent un séjour stationnaire avec des activités plus douces, voire des passe-temps peu sportifs : dominos, cartes et alcool. Il manque ici des études de cas géographiques pour restituer le fonctionnement de ces communautés éphémères que sont les groupes et les bases.

Le lecteur de Club Red se trouve ainsi dans une histoire du quotidien à la Michel de Certeau, mais aussi du contrôle des corps cher à Michel Foucault - deux auteurs utilisés ici, avec d'autres dont le sociologue Jean-Didier Urbain. Ce qui vaut pour les sociétés occidentales est-il directement transposable au contexte soviétique? Oui, semble affirmer Koenker, même s'il reste une spécificité dans l'accent mis sur l'égalité entre les participants, véritable "collectivisme socialiste en action » (p. 221) : en URSS, il s'agit d'une injonction officielle. Les conclusions de l'ouvrage paraissent toutefois faibles au regard de la masse documentaire utilisée. Il s'attache à démontrer la "réalité de la distinction socialiste» (p.147), palpable dans une société où les lieux de villégiature sont classés en une hiérarchie quasiment immuable des années 1930 aux années 1980 : au sommet Sotchi, puis la Crimée et les villes thermales du Nord Caucase. Le tourisme est vu comme un moyen pour l'intelligentsia au sens large de profiter de son pouvoir d'achat et de s'évader du quotidien. Reprenant les analyses classiques, depuis Vera Dunham (1976), sur l'accord tacite passé entre le régime et la "classe moyenne", Koenker s'enferme parfois dans une approche trop occidentalo-centrée: signe de ce déséquilibre, le dernier chapitre se contente en moins de vingt pages de montrer que le tourisme soviétique s'est «modernisé " pour devenir, dans les dernières décennies du régime, une « industrie» (p. 263). Le décret de 1969 sur le développement de ce secteur constitue pourtant une charnière douteuse de ce point de vue. Certes, avec la massification du milieu des années 1970, les citoyens sont désormais à même de choisir 
leurs destinations. Plusieurs sondages indiquent qu'ils adhèrent majoritairement au modèle des vacances en famille. Koenker parle de " convergence » (p. 273) du tourisme avec les standards (occidentaux, même s'ils sont inspirés des stations balnéaires bulgares et roumaines) des séjours de repos : chambres confortables, offre d'activités pour tous les âges, etc. Le pacte tacite conclu par le régime brejnévien avec une partie de la population repose ainsi, entre autres, sur sa capacité à répondre à cette demande : il y aurait un parallèle à faire avec l'expansion des infrastructures pour touristes fortunés lancée sous Vladimir Putin en prévision des Jeux olympiques d'hiver de 2014.

Club Red n'est pas un livre aisé à lire : la démonstration s'appuie sur une succession d'exemples vivants, mais dont l'articulation n'est pas toujours évidente. À vouloir trop embrasser, le récit s'égare parfois en anecdotes et en considérations accessoires qui font perdre de vue les enjeux posés au départ. Une difficulté supplémentaire tient à la faculté de l'auteure de synthétiser au maximum l'information abondante fournie par ses sources: les notes de bas de page sont longues et comptent plusieurs références d'archives et d'ouvrages, au point qu'il manque parfois la date précise d'un fait ou d'une citation. Sa tendance au discours indirect libre laisse un doute sur la distance observée à l'égard de certains discours, notamment lorsqu'il s'agit de critiques formulées dans les journaux, les livres d'or, ou des rapports internes des années 1930. D'autres sources moins officielles (entretiens, Mémoires, œuvres littéraires) auraient $\mathrm{pu}$ rééquilibrer certains jugements. Pour "expliquer la relation entre l'État et son peuple autant que la résilience du régime communiste et de ses valeurs » (p. 2), il aurait fallu dépasser davantage le constat des multiples pénuries (en termes de places de train, de logements pour famille, de matériel de randonnée ou de camping, de nourriture, etc.), pour mieux interroger le sens profond de ces séjours, périples et excursions du week-end qui ont forgé plusieurs générations de citadins soviétiques. S'agissait-il d'un passe-temps ou d'une raison de vivre alternative au projet officiel? Tout en s'attaquant au «paradoxe fondamental» des vacances en URSS - qu'un État autoritaire encourage «l'autonomie individuelle» (p. 7) -, Koenker prête finalement peu d'attention à l'impact des vacances en termes de fabrique d'un patriotisme localisé, ce à quoi appelle explicitement le décret de 1969 lorsqu'il parle de renforcer chez les citoyens d'URSS «l'amour du pays natal et la loyauté » (p. 275). Plus d'un kurort est devenu, comme Sotchi l'illustre à l'extrême avec ses bâtiments de style stalinien qui servirent de décor à tant de films y compris sous le dégel (La prisonnière du Caucase, etc.), un lieu commun de l'identité nationale. Voulu entre autres comme un moyen de connaître et d'aimer sa "patrie", le tourisme a-t-il rempli cet objectif de façon différenciée selon les catégories sociales et les époques? Sa dimension paramilitaire aurait pu être plus longuement analysée, de même que son impact en termes de construction d'un patrimoine national qui, outre les deux capitales Moscou et Leningrad (Saint-Pétersbourg), des ex-républiques baltes à la mythique Crimée et à la légendaire " route militaire " géorgienne, ou à l'ossétienne, continue de faire l'actualité de la Russie en ce début de xxI ${ }^{\mathrm{e}}$ siècle. Il manque enfin des cartes pour apprécier l'extension des pratiques, suivant les types de séjours et les catégories sociales, et pour les croiser avec d'autres dynamiques: exode rural et collectivisation, déportations et camps, modifications de frontières, protection de l'environnement, etc. Elles auraient donné plus de poids au travail de Koenker, qui reste un jalon précieux dans une histoire encore peu explorée. Que des randonneurs marchent en 1968 sur les traces des partisans criméens ou que d'autres, en 1971, suivent à vélo la route empruntée par une division de leur ville pendant la Grande Guerre patriotique (p. 259), qu'à la même 
époque des vacanciers aspirent à un repos fait de farniente et de soirées dansantes, d'eau minérale et de redécouverte du patrimoine de la Russie ancienne et prérévolutionnaire, que des touristes volontaires participent à la «protection de la nature » de tel ou tel site remarquable : voilà qui n'est pas anodin pour comprendre la civilisation soviétique et ses multiples héritages.

\section{NOTES}

1. Anne E. Gorsuch, All This is your World : Soviet Tourism at Home and Abroad After Stalin. New York: Oxford University Press, 2011.Voir le compte rendu par Andreï Kozovoï dans Cahiers du Monde russe, 52 (4), 2011 [en ligne] http://monderusse.revues.org/7508.

2. Anne E. Gorsuch, Diane P. Koenker, eds., Turizm : The Russian and East European Tourist under Capitalism and Socialism, Ithaca - Londres: Cornell University Press, 2006. Voir les comptes rendus par Anne Kropotkine dans Cahiers du Monde russe, 48 (4), 2007 [en ligne] http:// monderusse.revues.org/6138 et par Igor Tchoukarine, Revue d'études comparatives Est-Ouest, 39 (1), 2008.

\section{AUTEURS}

\section{LAURENT COUMEL}

CERCEC, Paris - CEFR, Moscou 